\title{
Enhanced Image Capture Through Fusion
}

\author{
Peter J. Burt \\ Keith Hanna \\ Raymond J. Kolczynski \\ David Sarnoff Research Center
}

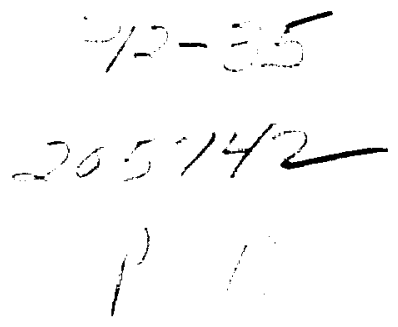

\begin{abstract}
Image fusion may be used to combine images from different sensors, such as IR and visible cameras, to obtain a single composite with extended information content. Fusion may also be used to combine multiple images from a given sensor to form a composite image in which information of interest is enhanced.

We present a general method for performing image fusion and show that this method is effective for diverse fusion applications. We suggest that fusion may provide a powerful tool for enhanced image capture with broad utility in image processing and computer vision.
\end{abstract}




\section{The Fusion Task}
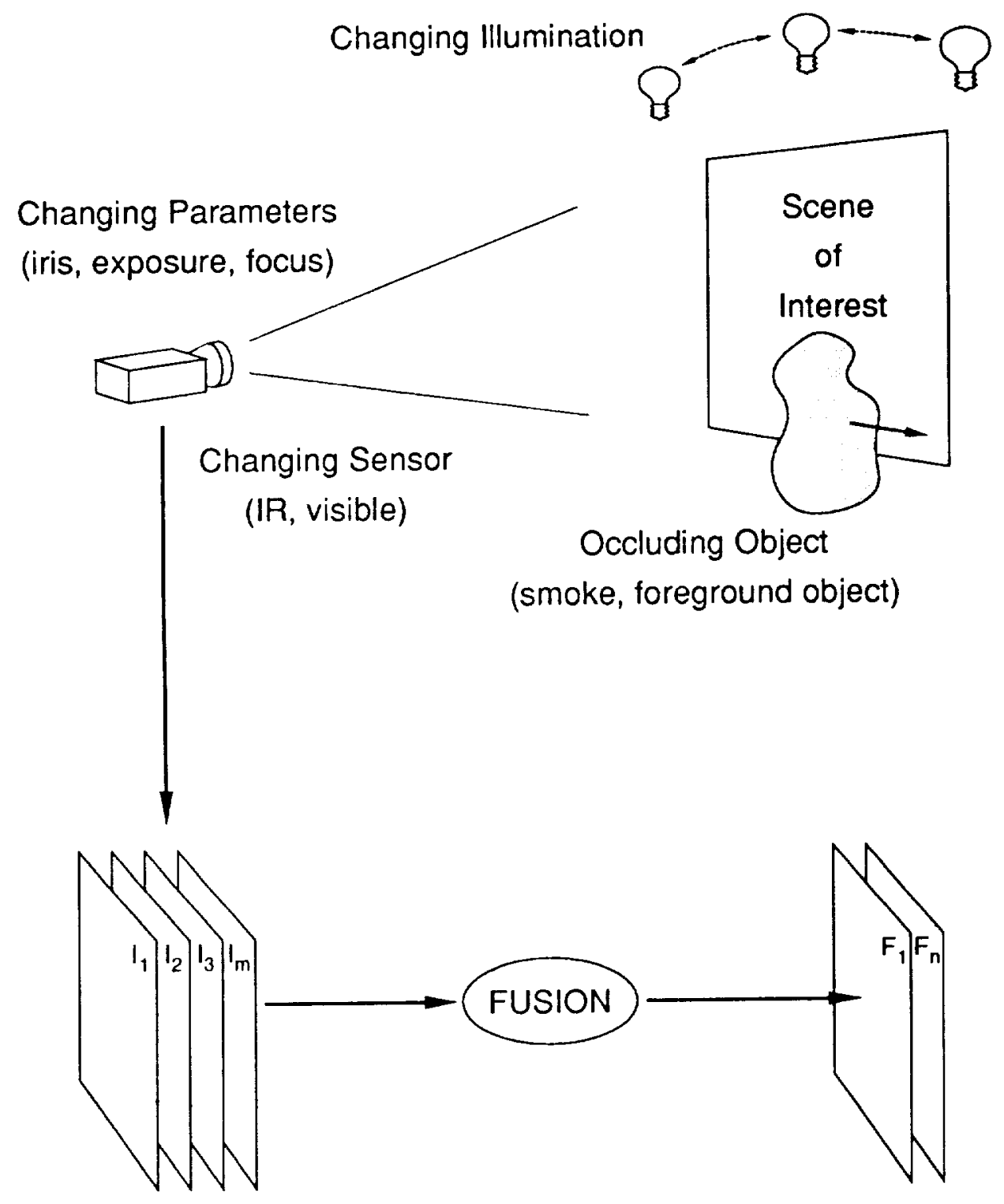

Set of Source Images

Set of Fused Images 


\section{Image Fusion: Objectives}

Combine two or more source images to obtain a single composite with extended information content.

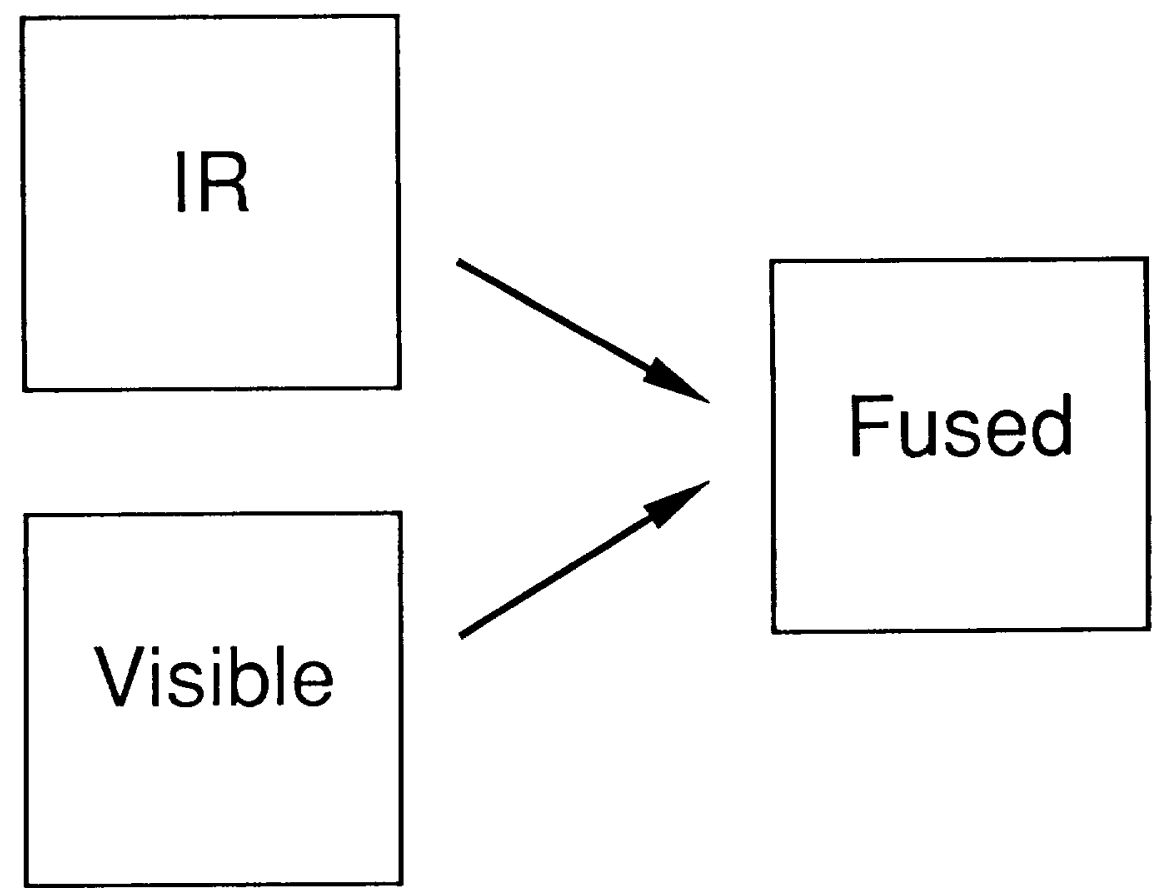

\section{Requirements}

- retain all useful information from the source images

- not introduce fusion artifacts into the combined image

- look "natural" 


\section{Technical Challenge}

Pixel averaging, results in ...
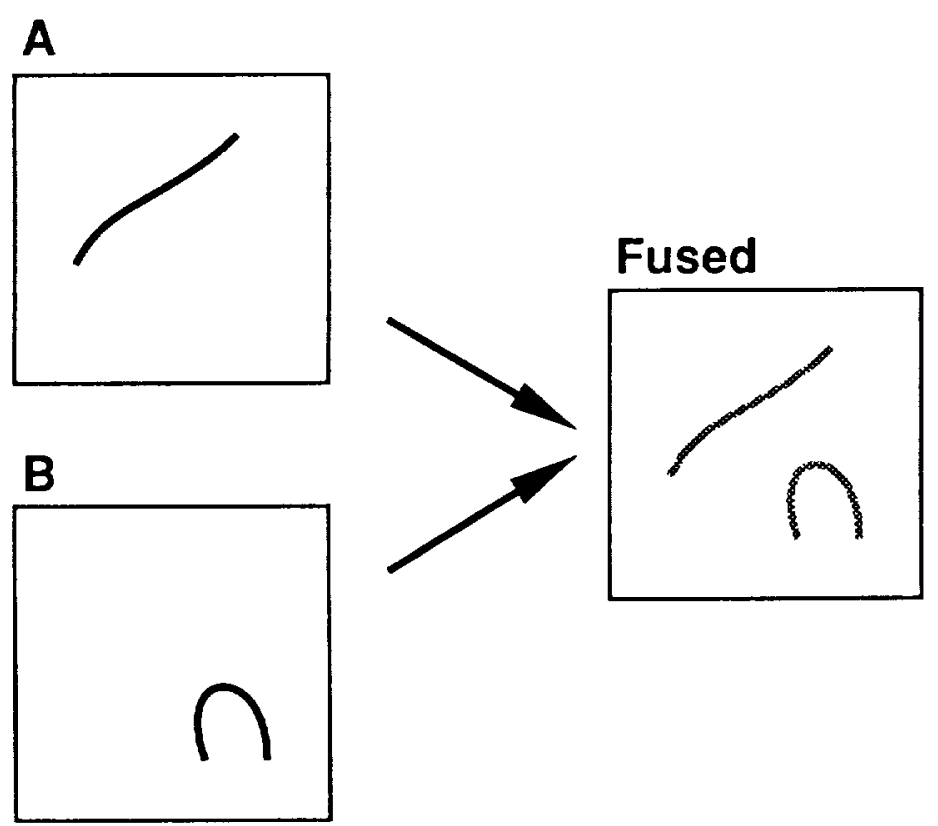

1. Loss of Contrast 


\section{Technical Challenge}

Pixel averaging, results in ...
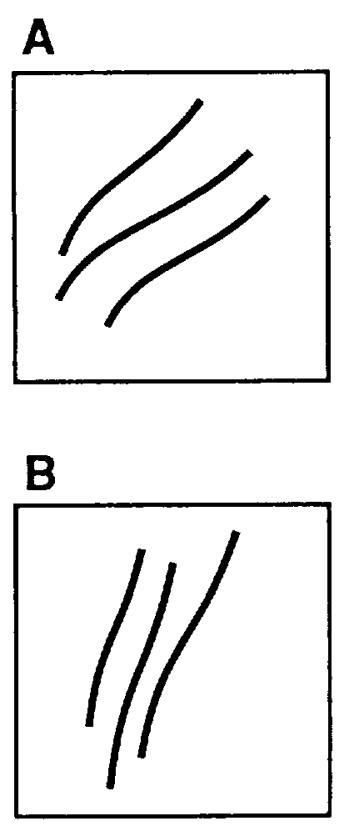

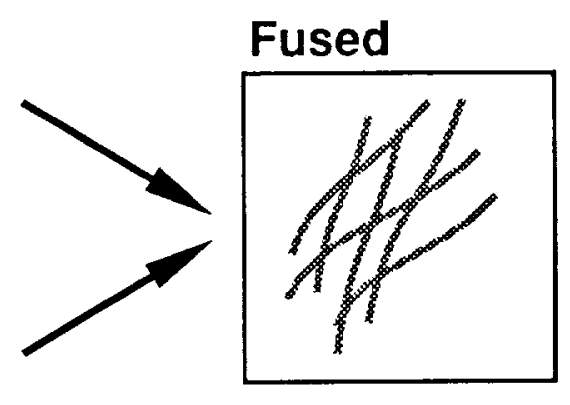

2. Double Exposure 


\section{Pixel-Based Approach}

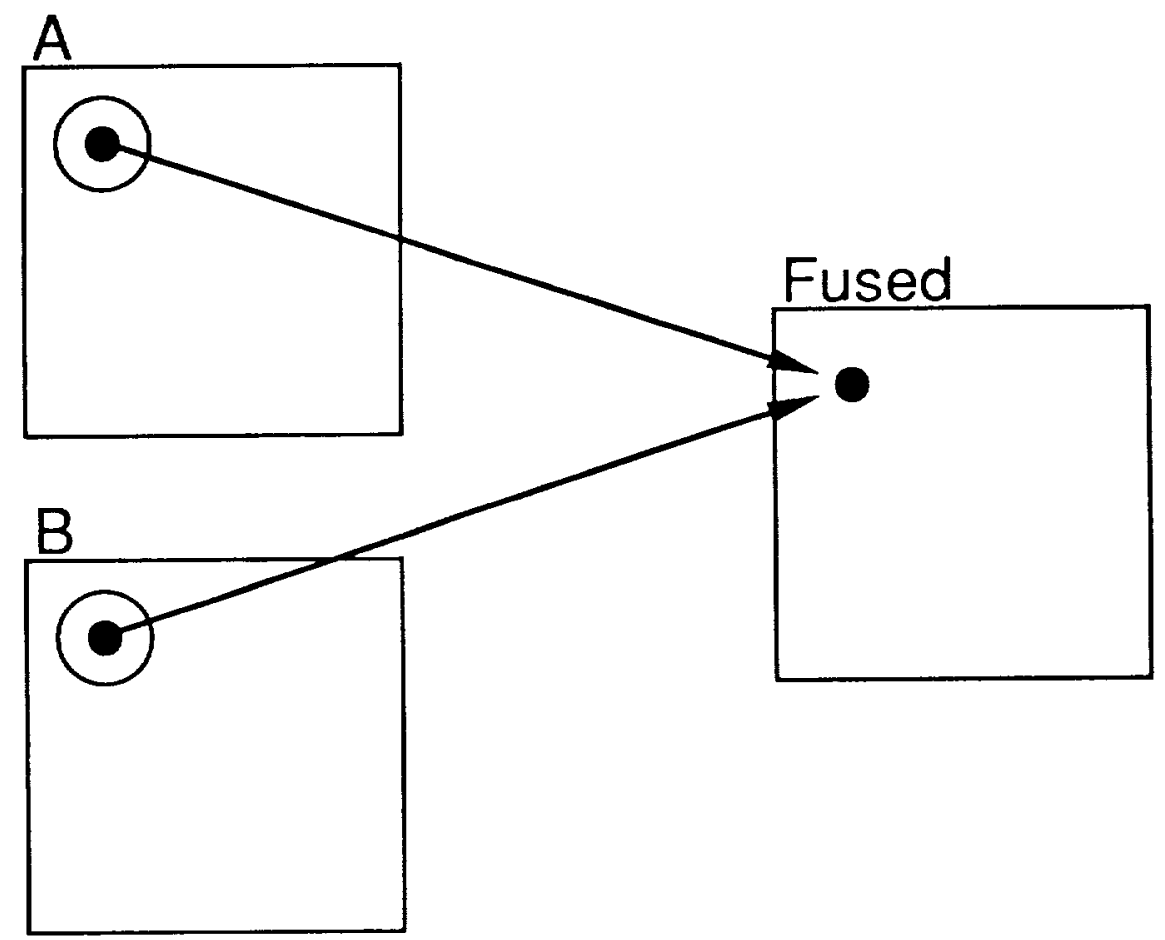

- each output pixel is computed separately

- based on the corresponding source image pixels

- or neighborhoods of corresponding pixels 


\section{Pattern-Selective Approach}

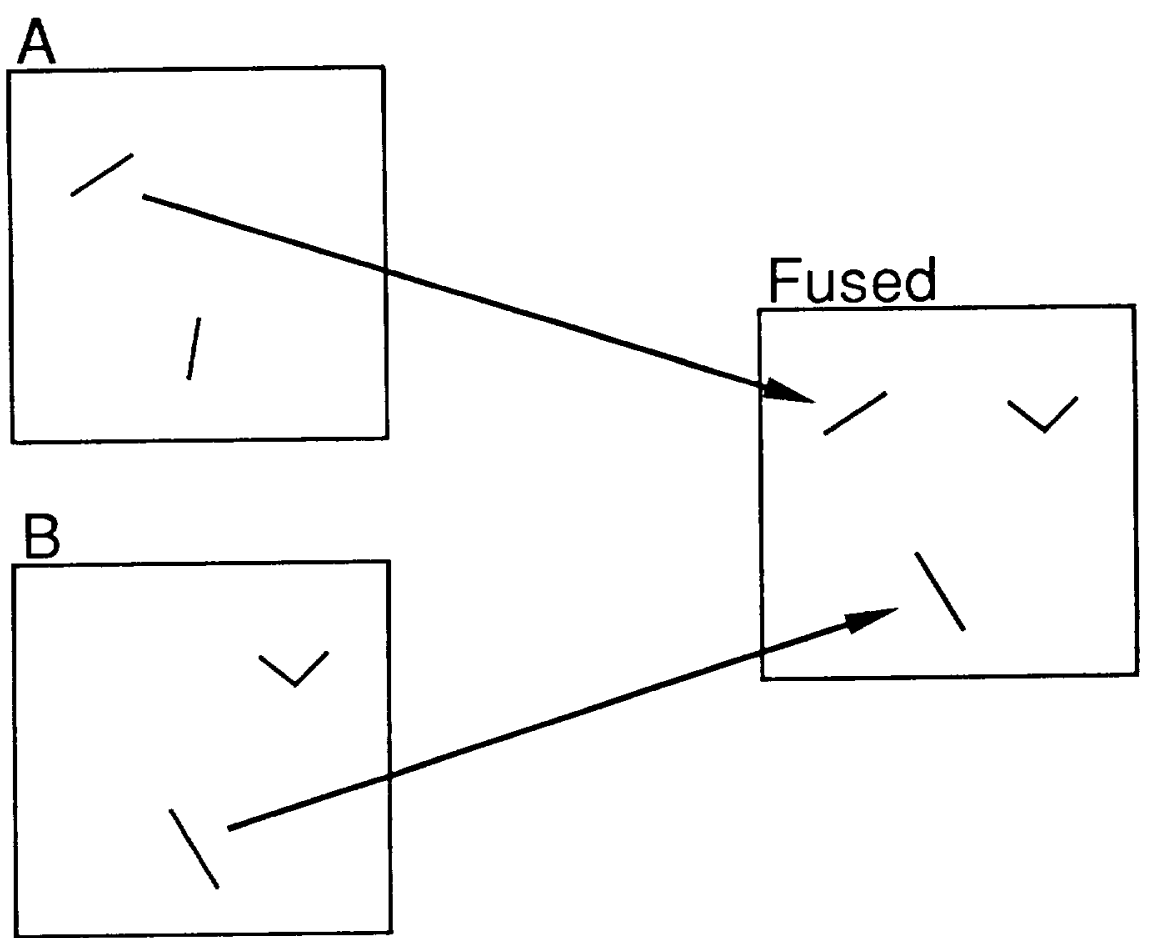

- copy a pattern at a time

- select most salient patterns only 


\section{Composite Imaging}

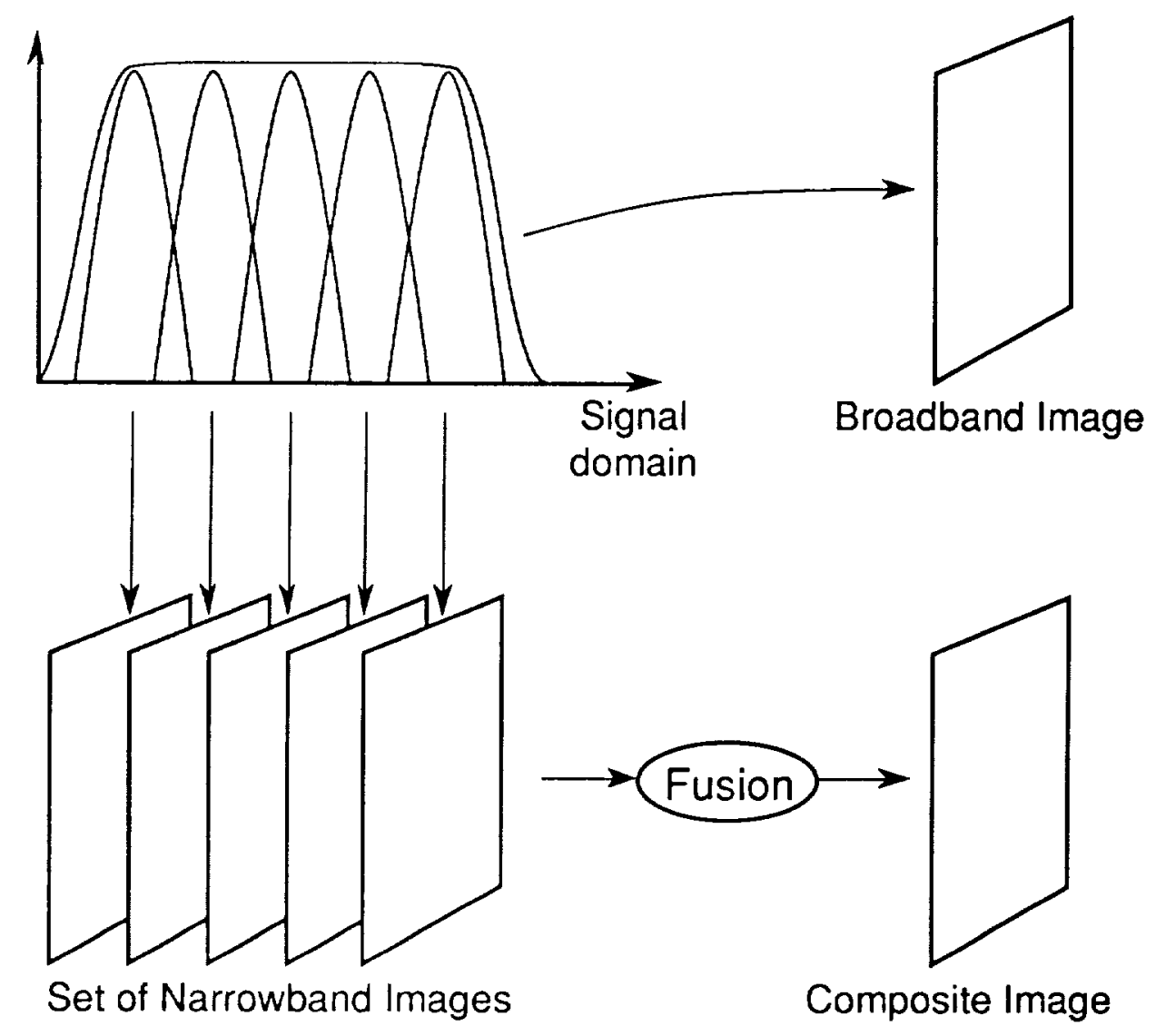




\section{Pyramid-Based Fusion: Some History...}

$\begin{array}{lll}1983 & \text { Burt } & \begin{array}{c}\text { model of human binocul } \\ \text { vision }\end{array} \\ 1984 & \text { Adelson } & \text { multi-focus } \\ 1990 & \text { Toet } & \text { IR and visual images } \\ 1991 & \text { Pavel,.. } & \text { noise model } \\ 1992 & \text { Tinkler } & \text { TI method }\end{array}$




\section{Laplacian Pyramid Transform}

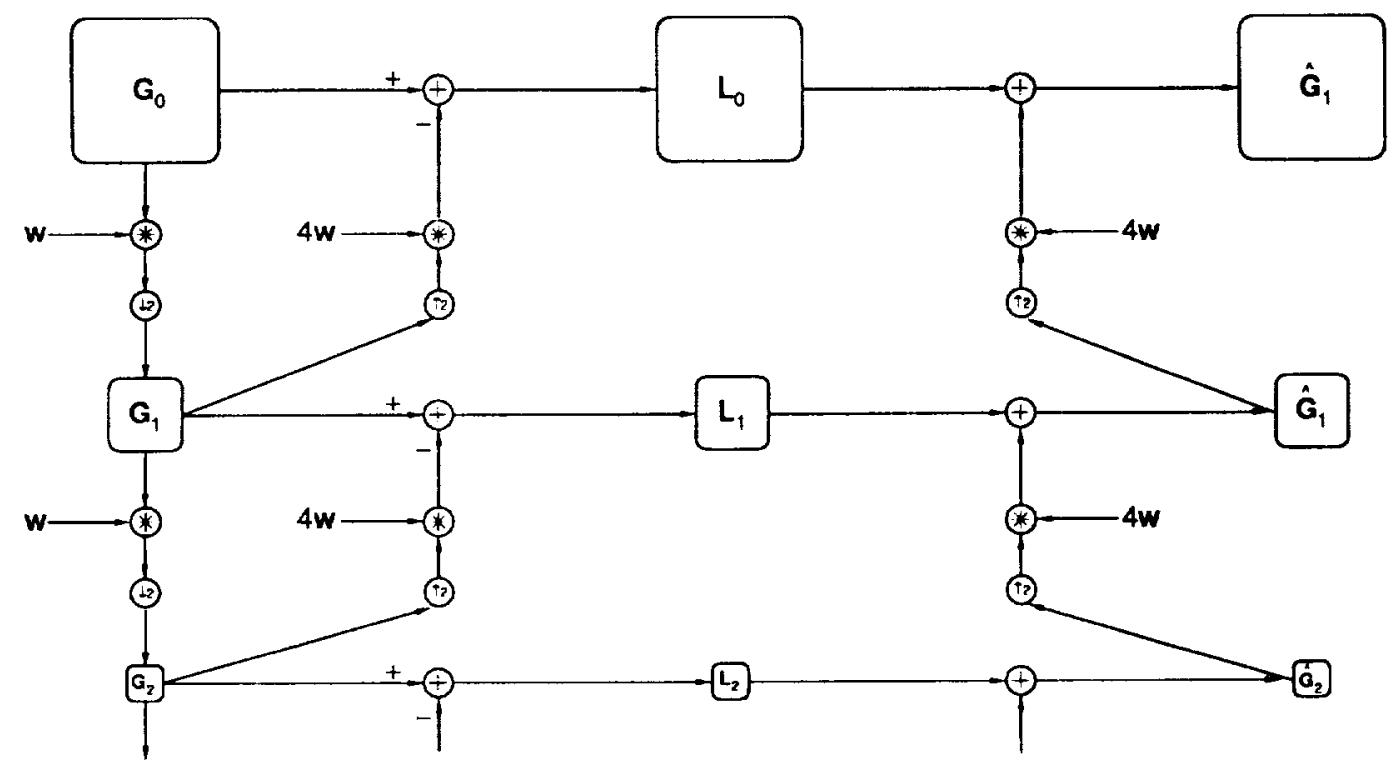

Gaussian

RE

Laplacian
Gaussian

Reconstructed 


\section{Gradient Pyramid Framework for Image Fusion}

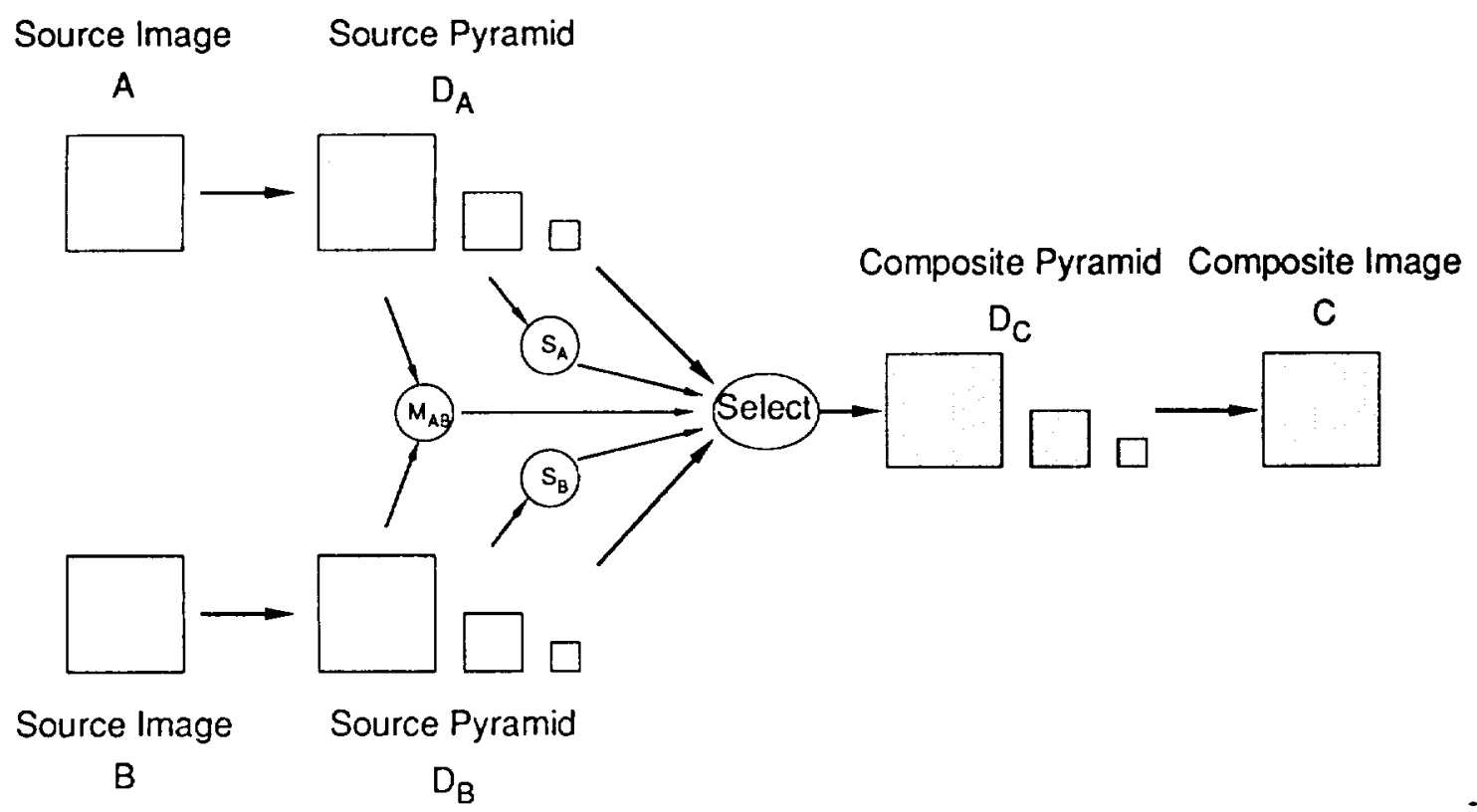


Composition Based on Scaled Relative Gradients

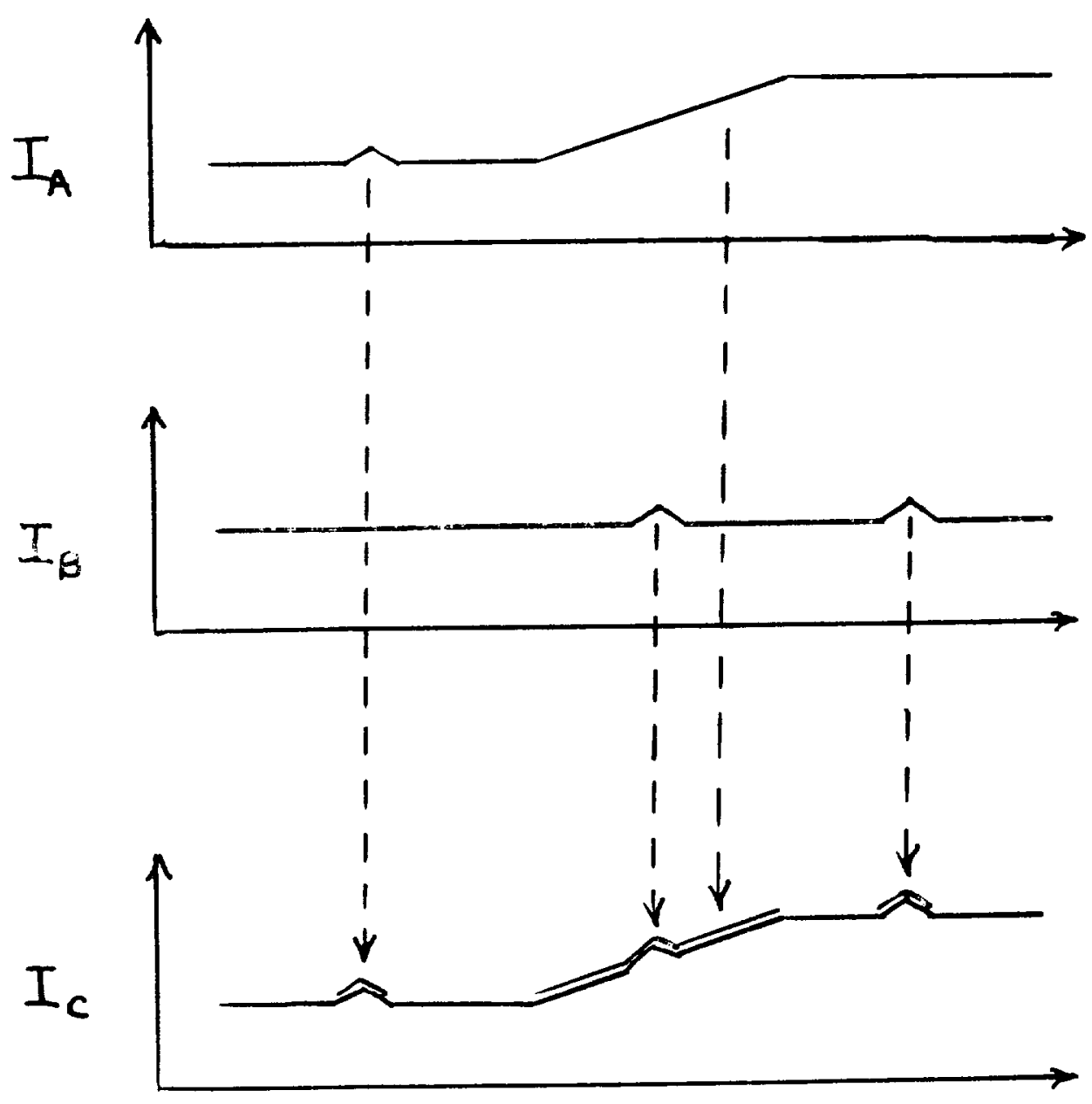

218 
Scaled Gradient Operators

$G(x, r)$

$\frac{d}{d x} G$
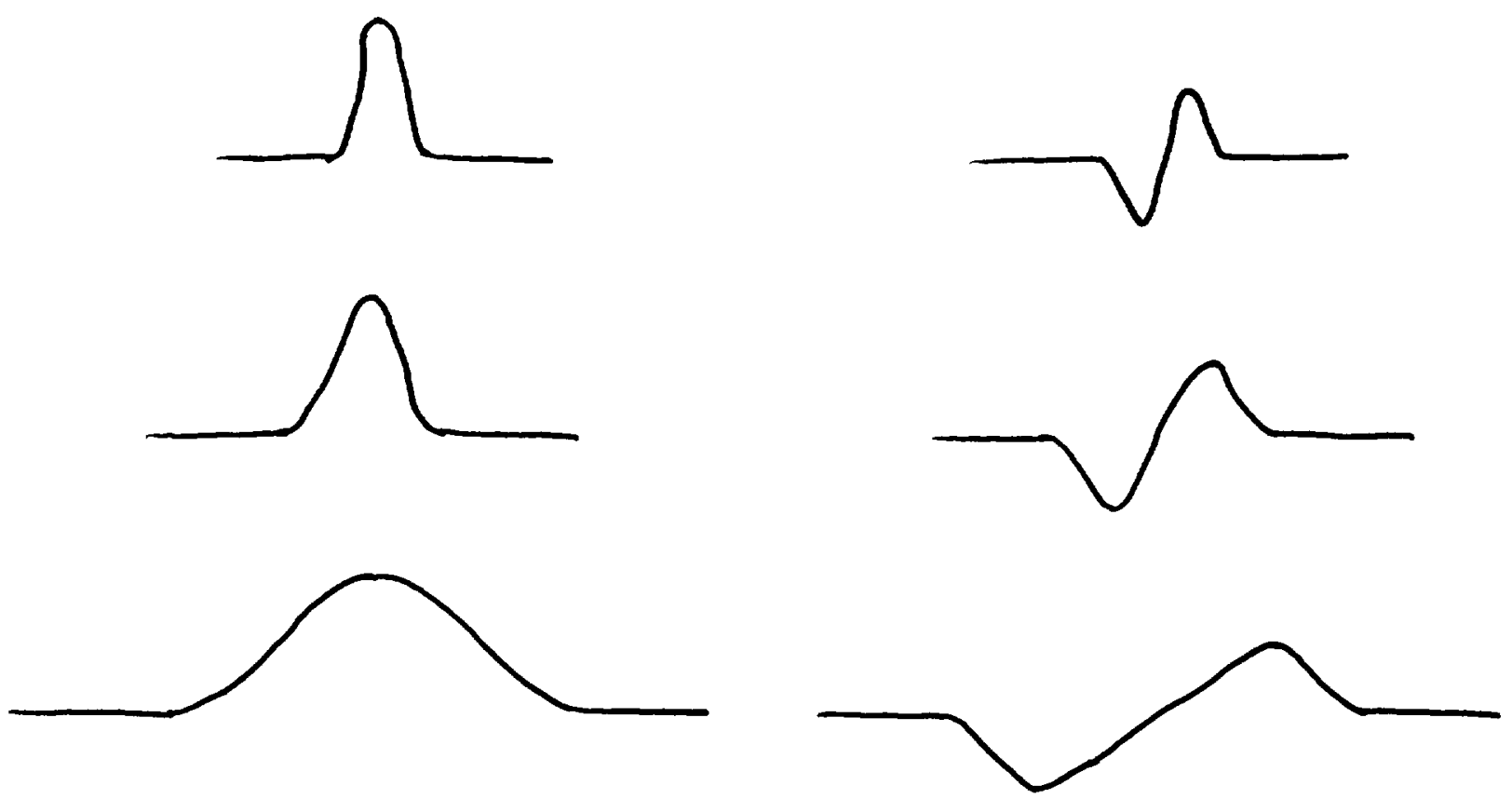

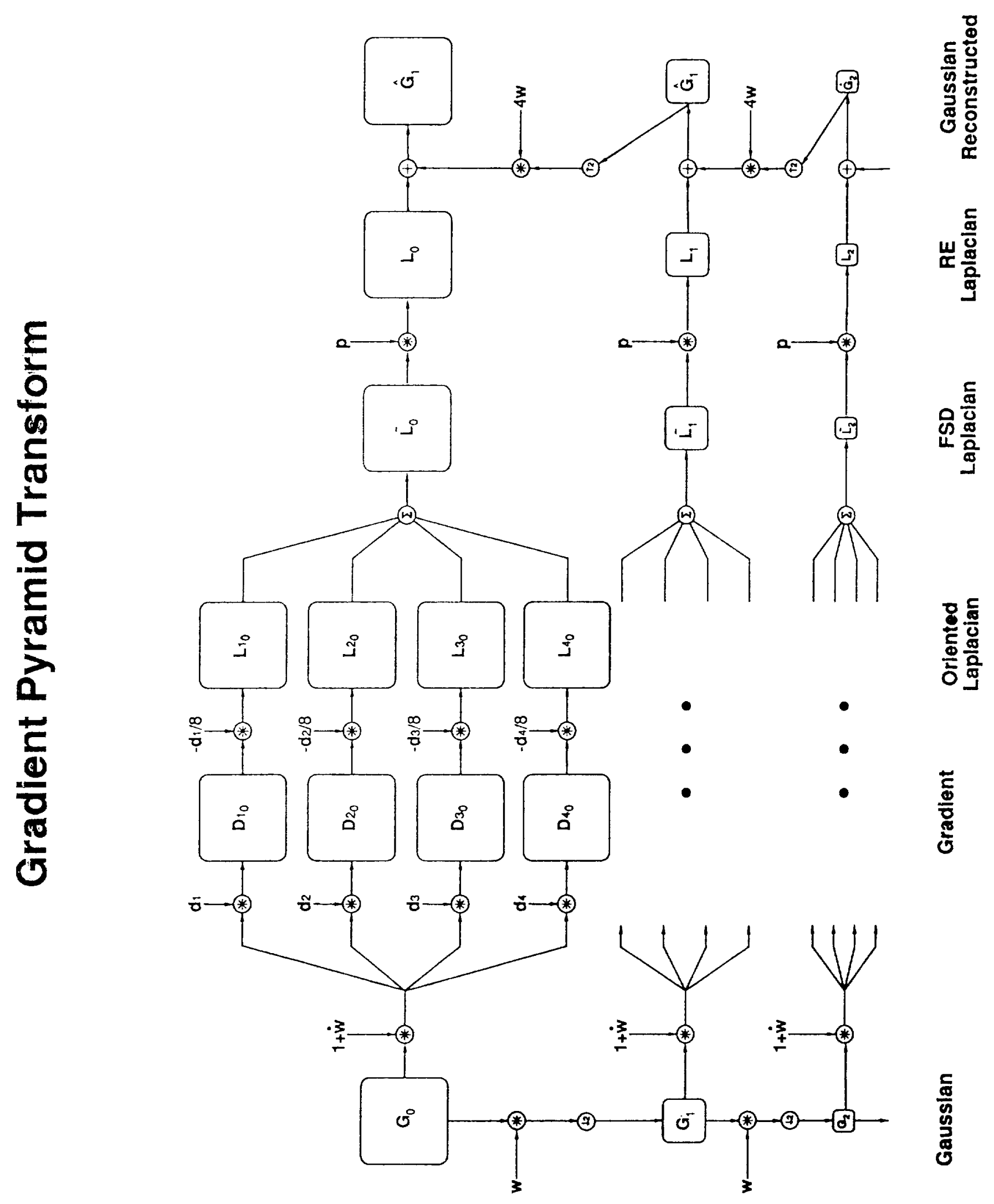

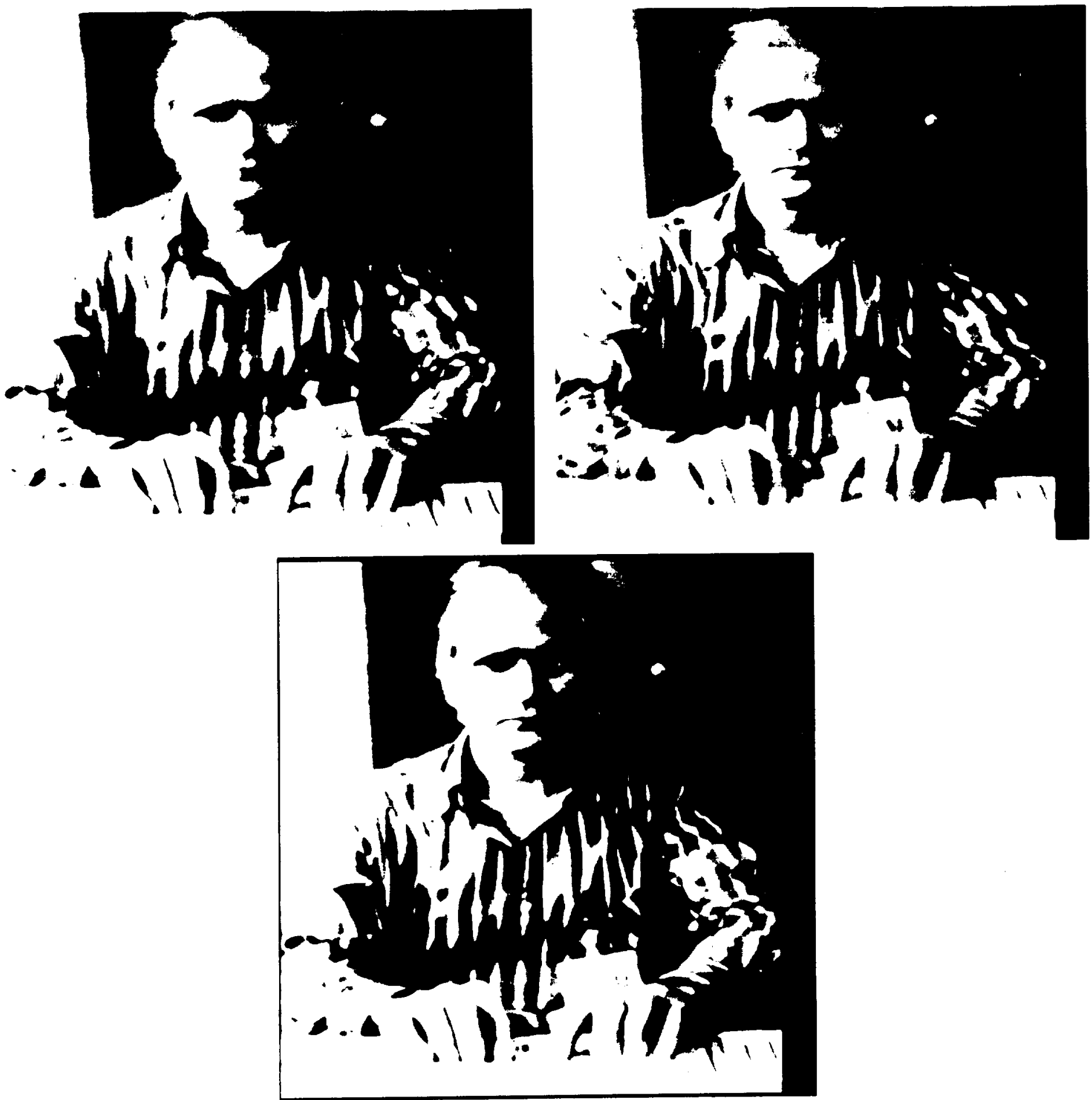

Multi-focus example of gradient pyramid fusion. ( $a$ and $b$ ) Source images obtained with a camera lens set to focus at different distances. (c) The fused image has an extended depth of field. 

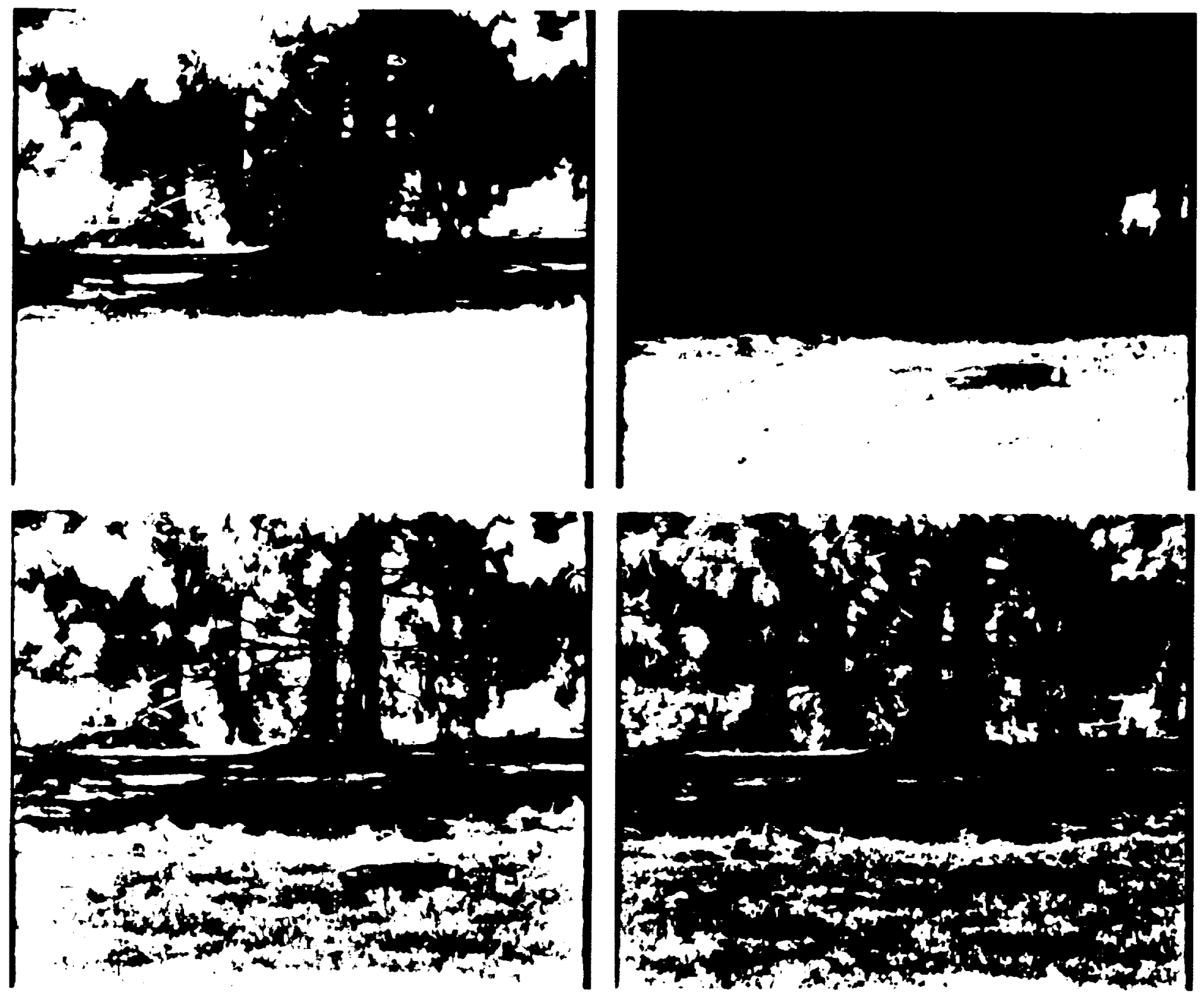

Multi-exposure example of gradient pyramid fusion. ( $a$ and $b$ ) Source images obtained with different camera exposure settings to observe patterns in shaded regions $(a)$ and bright, sun-lit regions $(b)$. (c) The fused image includes detail from both regions. (d) Pyramid samples values are normalized and quantized to just 4 bits to demonstrate that a broad dynamic range scene can be represented by a narrow dynamic range signal without loss of critical detail. 


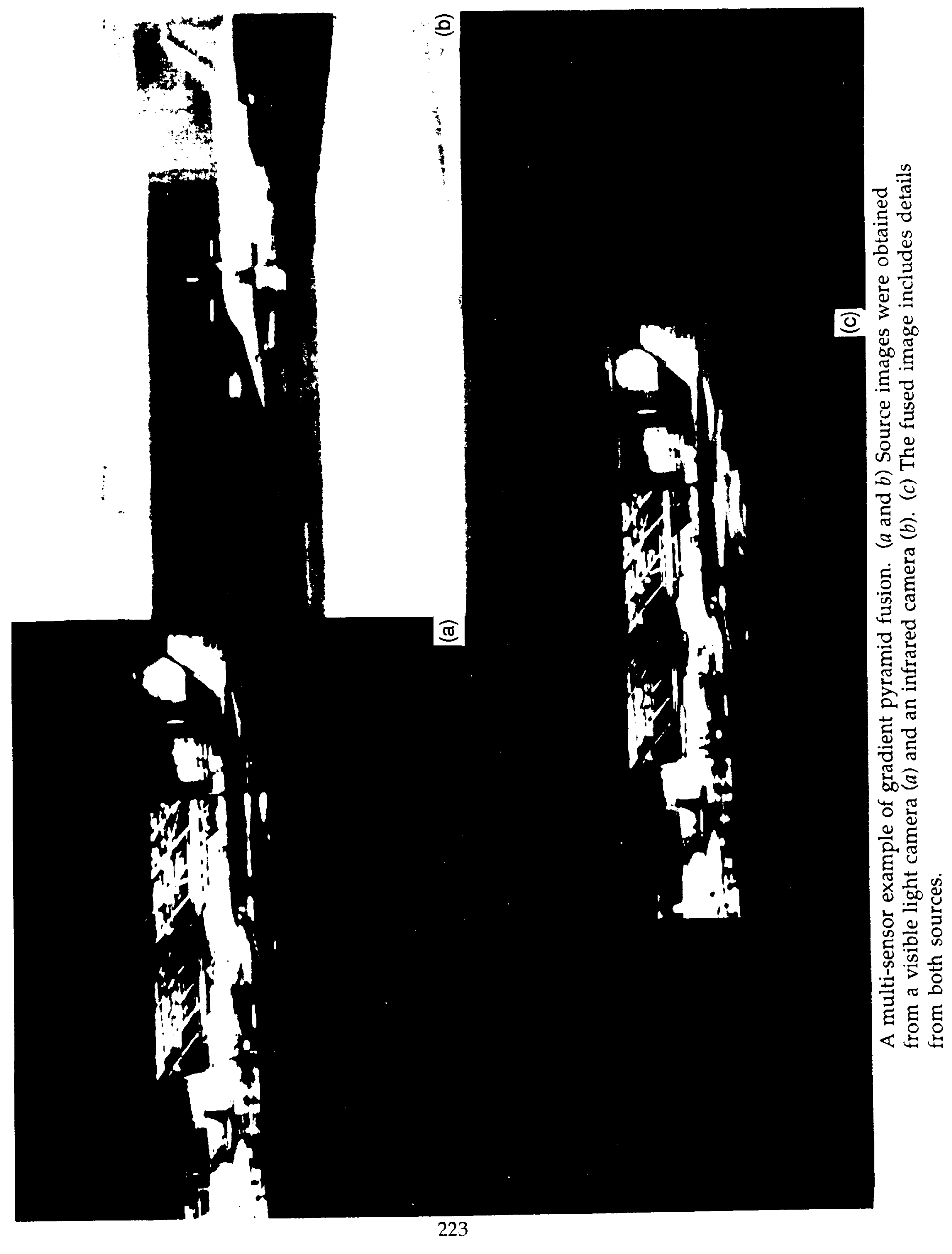




\section{Summary}

- Enhance image capture by combining observations

- Combine to preserve contrast (max gradient)

- Gradient pyramid framework (multiscale)

- Deliberately limit each observation (narrow band) 